\title{
Efecto del ácido abscísico sobre fenotipo y calidad del fruto en vid Shiraz
}

\section{Effect of abscisic acid on phenotype and fruit quality in Shiraz grapes}

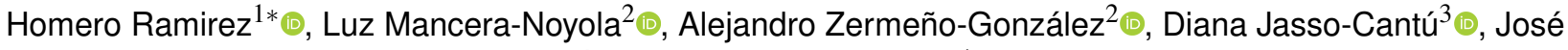 \\ Ángel Villarreal-Quintanilla ${ }^{4}$ \\ ${ }^{1}$ Departamento de Horticultura, Universidad Autónoma Agraria Antonio Narro, Calz. Antonio Narro 1923, CP. 25315, Saltillo, Coahuila, \\ México. \\ ${ }^{2}$ Departamento de Riego y Drenaje, Universidad Autónoma Agraria Antonio Narro, Calz. Antonio Narro 1923, CP. 25315, Saltillo, \\ Coahuila, México. \\ ${ }^{3}$ Departamento de Fitomejoramiento, Universidad Autónoma Agraria Antonio Narro, Calz. Antonio Narro 1923, CP. 25315, Saltillo, \\ Coahuila, México. \\ ${ }^{4}$ Departamento de Botánica, Universidad Autónoma Agraria Antonio Narro, Calz. Antonio Narro 1923, CP. 25315, Saltillo, Coahuila, \\ México. \\ *Autor de correspondencia: hrr_homero@hotmail.com
}

Nota científica recibida: 20 de septiembre de 2018 aceptada: 02 de noviembre de 2018

RESUMEN. El objetivo fue determinar el efecto del ácido abscísico (AAB) en el rendimiento y calidad de frutos de vid cultivar Shiraz. Se evaluó en campo la longitud y peso de racimos; y el rendimiento por planta. En laboratorio se determinó en los frutos maduros el pH, acidez y solidos solubles; además del contenido de polifenoles totales, procianidinas y antocianinas totales considerando que estas sustancias influyen en la coloración de los hollejos en las bayas. El experimento se realizó en Parras de la Fuente, Coahuila. El AAB se asperjó durante el envero a 0 (agua-testigo), 200, 400, 600 y $800 \mathrm{mg} \mathrm{L}^{-1}$. Se observó que el $\mathrm{AAB}$ a cualquier dosis no modificó parámetros fenotípicos; tampoco, solidos solubles, acidez y pH. El AAB provocó incrementos significativos en polifenoles $(23 \%)$, procianidinas $(25 \%)$ y antocianinas totales $(20 \%)$ en frutos con las dosis de 800,600 y $200 \mathrm{mg} \mathrm{L}^{-1}$, respectivamente.

Palabras clave: Antioxidantes, antocianinas, pigmentación, procianidinas.

ABSTRACT. The objective of this study was to determine the effect of abscisic acid (ABA) on the yield and quality of Shiraz cultivar grapes. Per-plant yield and bunch length and weight were evaluated in the field. In the laboratory the $\mathrm{pH}$, acidity and soluble solids in the ripe fruits were determined, as well as the content of total polyphenols, procyanidins and anthocyanins considering that these substances influence the coloring of the berry skins. The experiment was carried out in Parras de la Fuente, Coahuila. ABA was sprayed during veraison at 0 (water-control), 200, 400, 600 and $800 \mathrm{mg} \mathrm{L}^{-1}$. It was observed that ABA at any dose did not modify phenotypic parameters or soluble solids, acidity and $\mathrm{pH}$. ABA caused significant increases in total polyphenols $(23 \%)$, procyanidins $(25 \%)$ and anthocyanins $(20 \%)$ in fruits with doses of 800,600 and $200 \mathrm{mg} \mathrm{L}^{-1}$, respectively.

Key words: Antioxidants, anthocyanins, pigmentation, procyanidins.

\section{INTRODUCCIÓN}

México es de los principales exportadores de uva (SAGARPA 2017). En el 2016 la producción fue de 351309 t de las cuales el $23 \%$ se destino a la elaboración de vino, mientras que el resto se exporto a los mercados de Estados Unidos, Canadá y El Salvador (SIAP 2017). La industria vinícola requiere para su elaboración frutos con mayores niveles de pigmentación, aroma, grados brix y antioxidantes (McCormick 2012).

El cambio climático provoca adversidades en la fenología del fruto y reducción en la pigmentación de las bayas (Mirás-Ávalos 2017). Las fluctuaciones constantes de luz y temperatura degradan o inhiben la síntesis de antocianinas ocasionando una 
reducción de color en los frutos (Downey et al. 2006). El ácido abscísico (AAB) es una hormona que acelera la maduración en frutales (Rodríguez-Concepción y Boronat 2002). Con base a lo anterior, el objetivo fue evaluar el efecto del ácido abscísico sobre las características fenotípicas y la calidad en frutos de uva cultivar Shiraz.

\section{MATERIALES Y MÉTODOS}

La investigación se realizó en el 2017 en el viñedo San Lorenzo ubicado en Parras de la Fuente, Coahuila, México; ubicado a $30^{\circ} 30^{\prime} 0.33^{\prime \prime}$ LN y $102^{\circ}$ 11' 31.9" LO con altitud de 1505 msnm, el clima es BSO k(x') (e), con temperatura media anual de 14 a $18{ }^{\circ} \mathrm{C}$, precipitación de $366 \mathrm{~mm}$ y humedad relativa promedio de $51 \%$. Mientras que la segunda etapa se realizó en el laboratorio de postcosecha de la Universidad Autónoma Agraria Antonio Narro (UAAAN), ubicada en Saltillo, Coahuila, México. Se utilizaron plantas de vid del cultivar Shiraz, clon 174/S04 de 12 años, establecidas a 1.5 por $2.5 \mathrm{~m}$ con densidad de 2660 plantas ha $^{-1}$, el manejo del cultivo se realizó con el estándar de la empresa Casa Madero. Se evaluaron cuatro dosis de ácido abscísico (Protone $S G \AA 20 \%$ ) y un testigo (agua), realizando la aplicación en la etapa de envero. Las concentraciones de $A A B$ de $200,400,600$ y $800 \mathrm{mg} \mathrm{L}^{-1}$ se asperjaron a los racimos a punto de goteo, con una aspersora manual de mochila de $10 \mathrm{~L}$ de capacidad. El experimento se estableció bajo un diseño de bloques completos al azar con cuatro repeticiones por tratamiento. Los análisis y la comparación de medias de Tukey ( $p$ $\leq 0.05$ ) se realizaron con el porgrama RStudio para Windows versión 10.

Se seleccionaron cuatro racimos por planta a los que se les midió la longitud del raquis con una cinta Staley, Power Lock 8m/26'. La cosecha se realizó a los 52 días después de la aplicación del AAB. Además del peso del racimo por medio del pesado de cada racimo para luego determinar el rendimiento por planta. Los racimos seleccionados se colocaron en bolsas de plástico en una hielera y trasladaron al laboratorio de postcosecha de la UAAAN para conservarse a temperatura de $-10{ }^{\circ} \mathrm{C}$ para los análisis posteriores.

La determinación de $\mathrm{pH}$, acidez y solidos solubles (SS) en frutos se realizó de un extracto de 10 a $15 \mathrm{~mL}$ de jugo por planta con la técnica de la AOAC (1990). El pH se midió con un potenciómetro marca Hanna Hi98130. La acidez se determinó por titulación con $\mathrm{NaOH}$ al $0.1 \mathrm{~N}$ y fenolftaleína al $1 \%$, expresada como porcentaje equivalente de ácido tartárico. Los SS se determinaron en un $1.0 \mathrm{~mL}$ de jugo con un refractómetro Atago ATC-E.

El contenido de polifenoles totales se determinó por el método de Wong-Paz et al. (2014). Para lo cual se tomaron $5 \mathrm{~g}$ de fruta en un matraz de 250 $\mathrm{mL}$ con $50 \mu \mathrm{L}$ de metanol al $80 \%$ colocados en baño ultrasónico (Brason B series 5510) para extraer la substancia activa, para luego filtrar en papel Whatman No. 1, y tomar una alícuota de $20 \mu \mathrm{L}$, que se mezclaron con $20 \mu \mathrm{L}$ del reactivo de Folin-Ciocalteu. Para luego agregar $20 \mu \mathrm{L}$ de carbonato de sodio (7.5 $\mathrm{p} / \mathrm{v}$ ) y diluir en $125 \mu \mathrm{L}$ de agua destilada para tomar la lectura en un espectrofotómetro a $790 \mathrm{~nm}$. El contenido de polifenoles totales se expresó en equivalentes de ácido gálico por gramo de peso seco, de acuerdo a la curva de calibración construida en un rango de 0 a $600 \mathrm{mg} \mathrm{L}^{-1}$.

Las procianidinas totales se determinaron con la técnica de HCl-butanol (Porter et al. 1986). Se pesó $1 \mathrm{~g}$ de muestra y se colocó en un tubo de ensayo con tapón de rosca, a los que se le agregaron $5 \mathrm{~mL}$ de metanol y dejar reposar por $12 \mathrm{~h}$ para luego filtrar con papel Whatman 1, del extracto se tomó una alícuota de $250 \mu \mathrm{L}$ que se transfirieron a un tubo de ensayo con $1.5 \mu \mathrm{L}$ de $\mathrm{HCl}$-butanol al $5 \%$ $(\mathrm{v} / \mathrm{v})$ y $50 \mu \mathrm{L}$ de reactivo férrico. La mezcla se calentó por $1 \mathrm{~h}$ en baño maría (Precisión Modelo 84) a temperatura de $90{ }^{\circ} \mathrm{C}$. La lectura se tomó con un espectrofotómetro Jenway modelo $6320 \mathrm{D}$ a longitud de onda de $550 \mathrm{~nm}$. Los datos obtenidos se expresaron como equivalentes del estándar de procianidina-B1 (Sigma-Aldrich $\left.{ }^{\circledR}\right)$ generado de una curva calibrada en el rango 0 a $600 \mathrm{mg} \mathrm{L}^{-1}$.

El contenido de antocianinas totales en los frutos de los tratamientos se obtuvo por medio de la técnica de espectrofotometría (AOAC 1990). Para lo cual se tomaron $2.5 \mathrm{~g}$ de hollejo de bayas, se incubaron 
por $24 \mathrm{~h}$ en solución extractora de $\mathrm{HCl} 3 \mathrm{~N}$ y metanol al $85 \%$, para luego almacenar a temperatura de $8{ }^{\circ} \mathrm{C}$ en oscuridad. La muestra se maceró, filtró y aforó a $100 \mathrm{~mL}$ con la solución extractora. Se tomaron $4 \mathrm{~mL}$ de muestra y transfirieron a una celdilla, a la que se le agregaron $2 \mathrm{~mL}$ de peróxido de hidrogeno al $30 \%$, la lectura se tomó con un espectrómetro a longitud de $520 \mathrm{~nm}$. Los resultados se obtuvieron con la formula:

Antocianinas $(m g 100 g)=$

$50 * \%$ absorbancia $525 \mathrm{~nm}$

solucion extractora $0.405 *$ peso de la muestra

\section{RESULTADOS Y DISCUSIÓN}

El $A A B$, en las dosis evaluadas no modificó la longitud y peso de racimos, ni afectó el rendimiento (Tabla 1). Pero las dosis de 600 y $800 \mathrm{mg} \mathrm{L}^{-1}$ incrementaron el peso de racimos y rendimiento en un 4.2 y $15.4 \%$ en comparación con el testigo; mientras el rendimiento incremento en un 3.7 y $24.9 \%$, respectivamente. Estos resultados coinciden con Peppi et al. (2006), quienes al aplicar AAB en la etapa de envero no observaron variación en el peso final de las bayas del cultivar Flame Seedless, debido a que este proceso esta relacionado con la división celular de los frutos, así como el contenido de solutos presentes.

$\mathrm{El} \mathrm{pH,contenido} \mathrm{de} \mathrm{ácido} \mathrm{tartárico} \mathrm{y} \mathrm{SS} \mathrm{no} \mathrm{se}$ modificaron con los tratamientos de AAB (Tabla 2); lo que coincide con lo reportado por Sandhu et al. (2011) quienes encontraron que el $A A B$ no altera el $\mathrm{pH}$ y contenido de $\mathrm{SS}$ de frutos de las variedades Noble y Alachua. El aumento de contenido de SS en la maduración se debe a la hidrólisis de los almidones a azucares simples (Aúlar et al. 2001) y su balance depende de las características metabólicas, $\mathrm{pH}$ y actividad enzimática del cultivar. Lo que influye en el ablandamiento y coloración de los frutos (Wills et al. 2007). El contenido de polifenoles totales aumentó de forma significativa $(p \leq 0.05)$ con las dosis de AAB (Figura 1). La concentración de $600 \mathrm{mg} \mathrm{L}^{-1}$ mostró el mayor incremento que fue superior en 130 $\mathrm{mg} \mathrm{L}^{-1}$ de polifenoles totales que el testigo. Lo que coincide con los resultados de Rufato et al. (2016), quienes reportan incrementos de $600 \mathrm{mg} \mathrm{L}^{-1}$ de $A A B$ en el cultivar Isabel, y con Jeon et al. (2004), quienes observaron que la síntesis de compuestos fenólicos aumenta con la aplicación de $A A B$ en él envero. El $A A B$ de cualquier dosis incrementó de forma significativa en el contenido de procianidinas (Figura 2), presentando los frutos tratados con $800 \mathrm{mg} \mathrm{L}^{-1}$ de $A A B$ el mayor contenido. Al respecto, Ramirez et al. (2018) reportan incrementos significativos en polifenoles al aplicar AAB en dosis de $200 \mathrm{a} 800 \mathrm{mg} \mathrm{L}^{-1}$ en el cultivar Cabernet Franc. Las procianidinas proporcionan astringencia e influyen de forma directa en el color del vino por su reacción covalente con antocianinas (Cassasa et al. 2015). Los tratamientos con AAB causaron incrementos superiores al $20 \%$ con respecto al testigo para el contenido de antocianinas; aunque entre ellos no hubo diferencias estadísticas (Figura 3). Los que coincide con Villalobos-Gonzalez et al. (2016), quienes observaron que el $A A B$ aplicado en dosis de $100 \mathrm{mg} \mathrm{L}^{-1}$ en el envero del cultivar Camenere incrementó cuatro veces el contenido de antocianinas en compración con el testigo. Al respecto, Peppi et al. (2006) reportan incrementos de la coloración de los frutos del cultivar Crimson con $400 \mathrm{mg} \mathrm{L}^{-1}$ de $A A B$, lo que atribuyen a la acumulación de antocianinas en la piel. Lo que se relaciona con el color de las uvas, que depende de la síntesis de antocianinas (Boss et al. 1996).

El ácido abscísico, aplicado en la etapa de envero, no afectó el pH, acidez y contenido de sólidos solubles de los frutos; ni la longitud del raquis, peso de racimos y rendimiento por planta. Pero las dosis de 200 a $800 \mathrm{mg} \mathrm{L}^{-1}$ tuvieron incrementos significativos en el contenido de polifenoles, procianidinas y antocianinas en los frutos. Lo que contribuye en la producción de frutos de mejor calidad para la elaboración de vinos. 
Ramirez et al.

Tabla 1. Efecto del ácido abscísico en la longitud del raquis, peso de racimo y rendimiento en el cultivar Shiraz.

\begin{tabular}{cccc}
\hline $\begin{array}{c}\text { Concentración de AAB } \\
(\mathrm{mg} \mathrm{L}-1)\end{array}$ & $\begin{array}{c}\text { Longitud del raquis } \\
(\mathrm{cm})\end{array}$ & $\begin{array}{c}\text { Peso de racimo } \\
(\mathrm{g})\end{array}$ & $\begin{array}{c}\text { Rendimiento por planta } \\
(\mathrm{kg})\end{array}$ \\
\hline 200 & $12.718^{a}$ & $177.187^{a}$ & $1.675^{a}$ \\
400 & $12.375^{a}$ & $197.187^{a}$ & $1.605^{a}$ \\
600 & $12.953^{a}$ & $186.25^{a}$ & $1.940^{a}$ \\
800 & $13.281^{a}$ & $206.25^{a}$ & $2.335^{a}$ \\
Testigo & $12.984^{a}$ & $178.75^{a}$ & $1.870^{a}$ \\
\hline Valores con la misma letra en columnas son estadísticamente iguales (Tukey, $\mathrm{p} \leq 0.05)$.
\end{tabular}

Tabla 2. Efecto del ácido abscísico en el pH, acidez y solidos solubles en frutos del cultivar Shiraz.

\begin{tabular}{cccc}
\hline $\begin{array}{c}\text { Concentración de ABA } \\
\left(\mathrm{mg} \mathrm{L}^{-1}\right)\end{array}$ & $\begin{array}{c}\mathrm{pH} \\
\mathrm{ns}\end{array}$ & $\begin{array}{c}\text { Acido tartárico } \\
(\%) \mathrm{ns}\end{array}$ & $\begin{array}{c}{ }^{\circ} \text { Brix } \\
(\%) \mathrm{ns}\end{array}$ \\
\hline 200 & $4.180^{a}$ & $0.390^{a}$ & $25.450^{a}$ \\
400 & $4.135^{a}$ & $0.365^{a}$ & $25.525^{a}$ \\
600 & $3.975^{a}$ & $0.423^{a}$ & $23.975^{a}$ \\
800 & $4.165^{a}$ & $0.348^{a}$ & $25.400^{a}$ \\
Testigo & $4.073^{a}$ & $0.355^{a}$ & $25.525^{a}$ \\
\hline
\end{tabular}

ns: no significativo a una $\mathrm{p} \leq 0.05$. Valores con la misma letra en columnas son estadísticamente iguales (Tukey, $p \leq 0.05$ ).

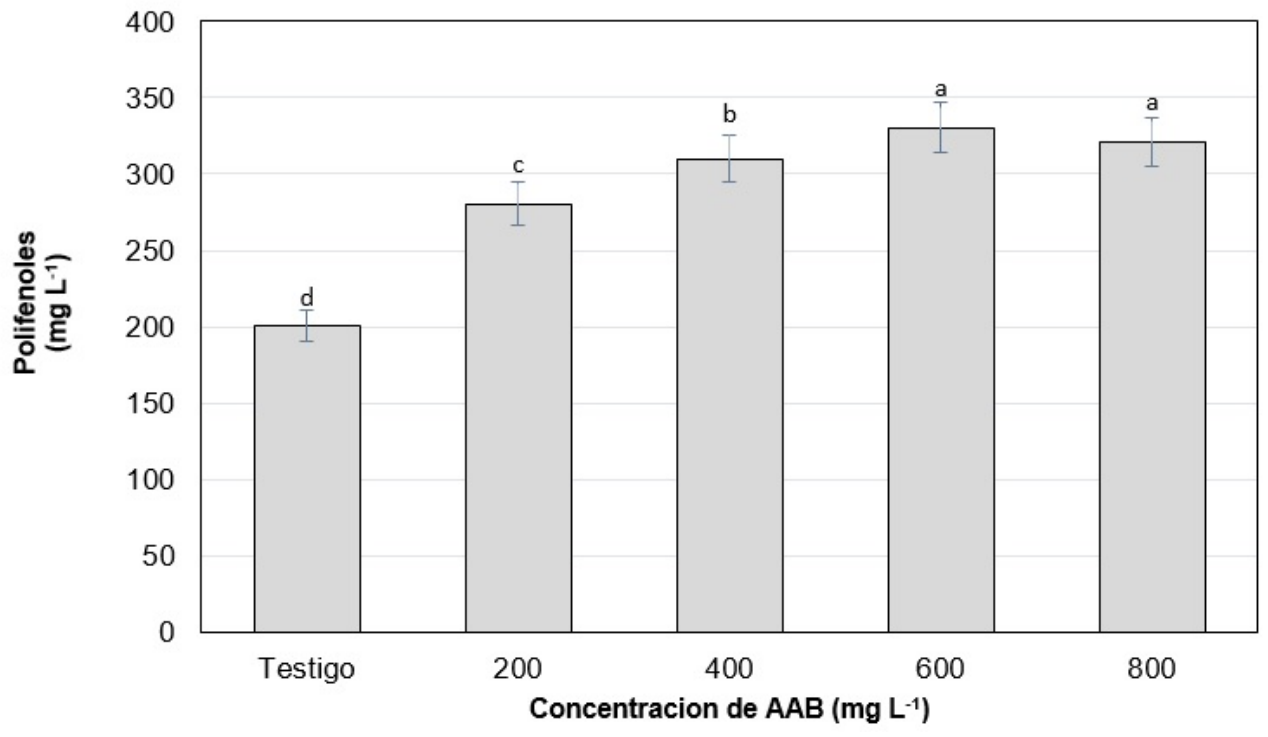

Figura 1. Efecto de ácido abscísico en el contenido de polifenoles totales en frutos maduros de vid en el cultivar Shiraz. Letras diferentes indican diferencia estadística (Tukey, $\mathrm{p} \leq 0.05$ ). 


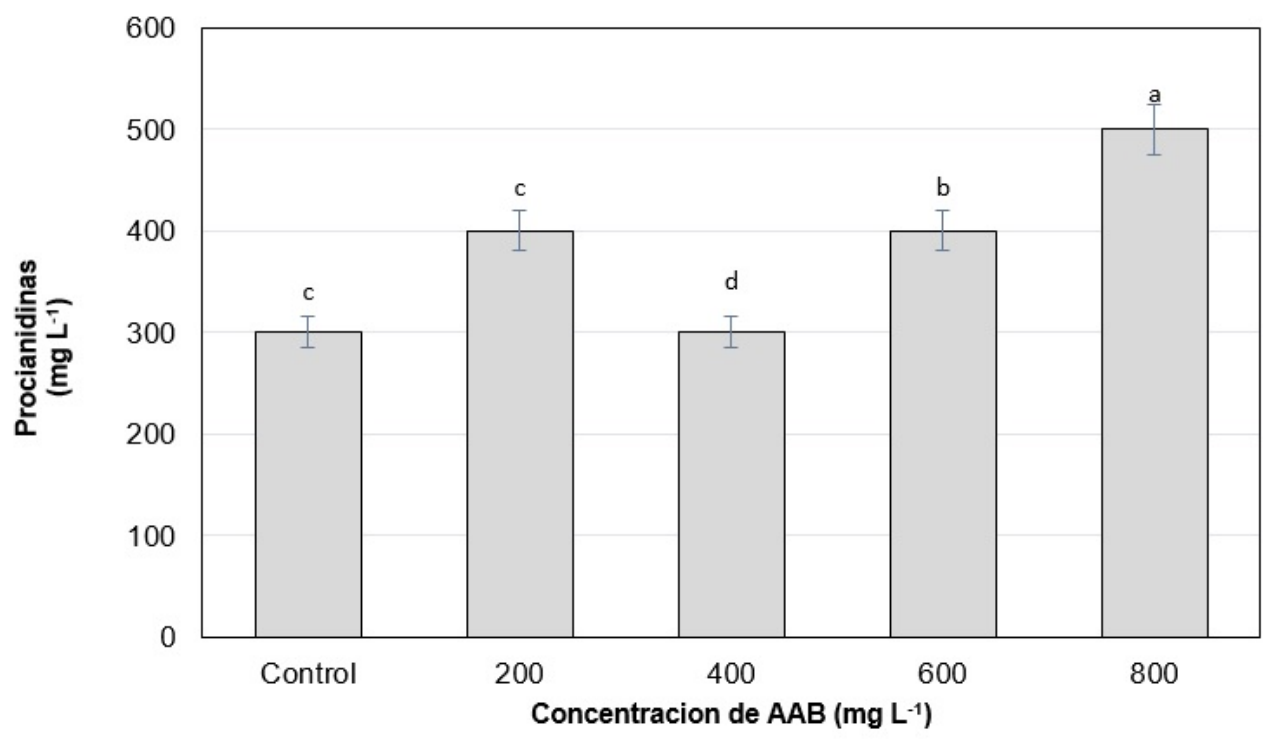

Figura 2. Efecto del ácido abscísico en el contenido de procianidinas en frutos maduros de vid en el cultivar Shiraz. Letras diferentes indican diferencia estadística (Tukey, $p \leq 0.05$ ).

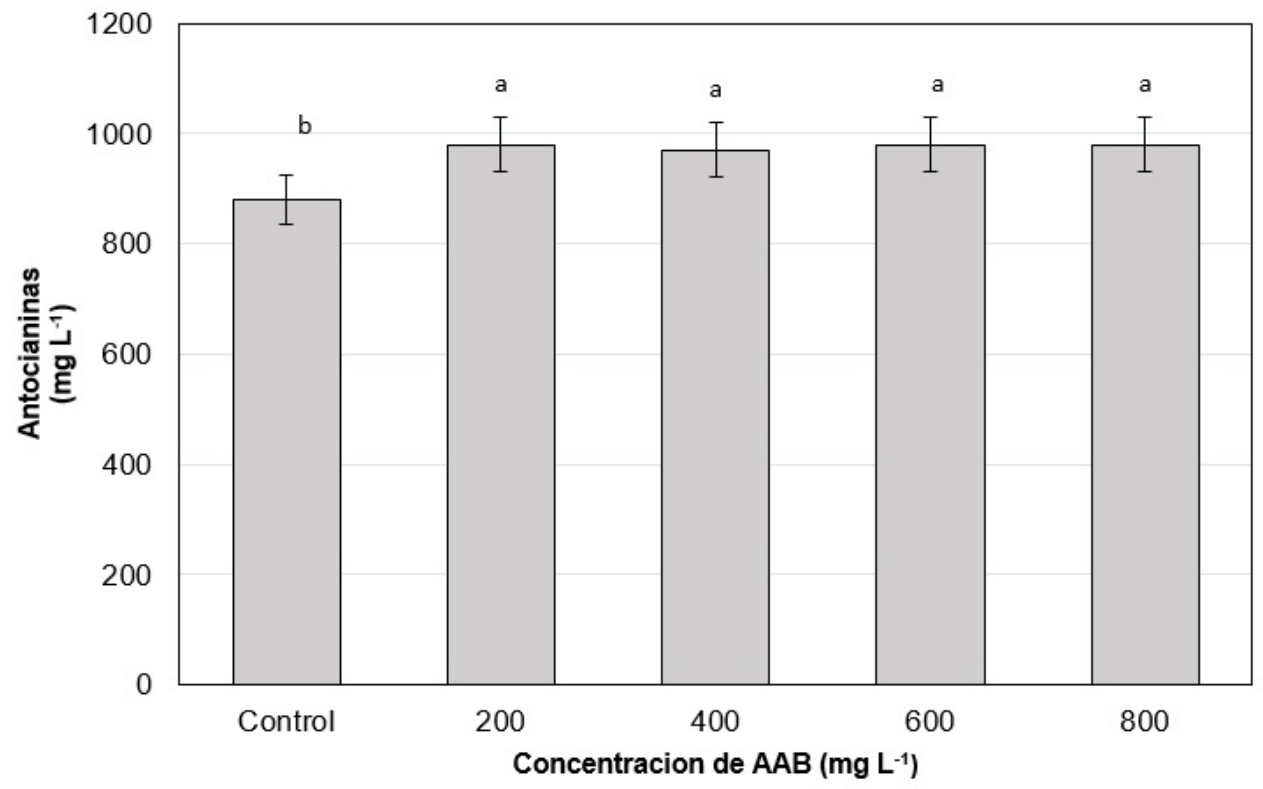

Figura 3. Efecto del ácido abscísico en el contenido de antocianinas en frutos maduros de vid en el cultivar Shiraz. Letras diferentes indican diferencia estadística (Tukey, $\mathrm{p} \leq 0.05$ ).

\section{LITERATURA CITADA}

AOAC (1990) Official Methods of Analysis. 15th ed. Association of Official Analytical Chemists. Arlington, USA. $66 \mathrm{p}$.

Aúlar J, Ruggiero C, Duringan J (2001) Efecto de la envoltura plástica y el tiempo de almacenamiento sobre el comportamiento postcosecha de frutos de parchita maracuyá. Bioagro 13: 15-21. 
Boss PK, Davies C, Robinson SP (1996) Analysis of the expression of anthocyanin pathway genes in developing Vitis vinifera cv. Shiraz grape berries and the implications for pathway regulation. Plant Physiology 111: 1059-1066.

Casassa L, Keller M, Harbertson J (2015) Regulated Deficit Irrigation Alters Anthocyanins, Tannins and Sensory Properties of Cabernet Sauvignon Grapes and Wines. Molecules 20: 7820-7844.

Downey MO, Dokoozlian NK, Krstic MP (2006) Cultural practice and environmental impacts on the flavonoid composition of grapes and wine. American Journal of Enology and Viticulture 57: 257-268.

Jeon ST, Goto-Yamamoto N, Kobayashi S, Esaka M (2004) Effects of plant hormones and shading on the accumulation of anthocyanins and the expression of anthocyanin biosynthetic genes in grape berry skins. Plant Science 167: 247-252.

McCormick RA (2012) A whole foods plant based health perspective, an opportunity for horticulture. Chronica Horticulturae 52: 5-9.

Mirás-Ávalos JM, Intrigliolo DS (2017) Grape composition under abiotic constrains: Water stress and salinity. Frontiers in Plant Science 8: Article 851. Doi: 10.3389/fpls.2017.00851.

Peppi MC, Fidelibus MW, Dokoozlian N (2006) El tiempo y la concentración de la aplicación de ácido abscísico afectan la firmeza, la pigmentación y el color de las uvas sin semilla. Scientia Horticulturae 41: 1440-1445.

Porter LJ, Hritsch LN, Chan BG (1986) The conversion of procyanidins and prodelphinidins to cyanidin and delphinidin. Phytochemistry 25: 223-230.

Ramírez H, Mancera-Noyola M, Zermeño-Gonzalez A, Jasso-Cantú D, Villarreal-Quintanilla JA (2018) Effect of abscisic acid on yield, anthocyanins, polyphenols and procyanidins in grape cv. Cabernet Franc (Vitis vinífera L). International Journal of Plant \& Soil Science 24: 5-7.

Rodríguez-Concepción M, Boronat A (2002) Elucidation of the methylerythritol phosphate pathway for isoprenoid biosynthesis in bacteria and plastids. A metabolic milestone achieved through genomics. Plant Physiology 130: 1079-1089.

Rufato L, Lerin S, Allebrandt R, Fagherazzi AF, Mario AE, Boff CE, et al. (2016) Abscisic acid applications increase color in grapes and juice of Isabel. Acta Horticulturae 1115: 217-223.

SAGARPA (2017) Planeación agrícola nacional 2017-2030. Coahuila, México https://www.gob.mx/cms/uploads/ attachment/file/257085/Potencial-Uva.pdf. Fecha de consulta: 12 de octubre del 2018.

Sandhu AK, Gray DJ, Lu J, Gu L (2011) Effects of exogenous abscisic acid on antioxidant capacities, anthocyanins, and flavonol content of Muscadine. grape (Vitis rotundifolia) skins. Food Chemistry 126: 982-988.

SIAP (2017) Producción agrícola nacional, uva mexicana. Coahuila, México https://www.gob.mx/cms/uploads/ attachment/file/257085/Potencial-Uva. Fecha de consulta: 12 de octubre del 2018.

Villalobos-González L, Peña-Neira A, Ibáñez F, Pastenes C (2016) Long-term effects of abscisic acid (AAB) on the grape berry phenylpropanoid pathway: Gene expression and metabolite content. Plant Physiology and Biochemistry 105: 213-223.

Wills RD, MacGlasson B, Graham D, Joyuce D (2007) Postharvest an introduction to the physiology and handling of fruit, vegetables and ornamentals. 5th Edition. CABI International. Wallingford, Reino Unido. 227p

Wong-Paz JE, Muñiz-Márquez DB, Aguilar-Zarate P, Rodríguez-Herrera R, Aguilar NC (2014) Microplate quantification of total phenolic content from plant extracts obtained by conventional and ultrasound methods. Phytochemical Analysis 25: 1-6. 